$\underline{\mathbf{P}-76}$

\title{
Quantification of Madecassoside, Asiaticoside, Madecassic Acid and Asiatic Acid in Centella Asiatica by Reverse Phase HPLC
}

\author{
Munira Mohd Amin Sharifuldin, Abdalrahim F.A. Aisha and Zhari Ismail* \\ Department of Pharmaceutical Chemistry, School of Pharmaceutical Sciences, Universiti Sains Malaysia, Minden \\ 11800, Pulau Pinang, Malaysia; E-mail: zhari@usm.my
}

Centella asiatica Linn. (Umbelliferae) or Pegaga has been widely used and studied as a wound healing, memory enhancer and anti-ageing agent. This plant contains high concentration of triterpene acids and saponins. This study aims to develop a reverse phase HPLC method for quantification of asiaticoside, madecassoside, asiatic acid and madecassic acid in fifteen different samples of $C$. asiatica extracts. The method developed was validated at $200 \mathrm{~nm}$ for selectivity, linearity, precision, accuracy and limits of detection and quantification. Selectivity was determined based on the retention time; linearity was in the range $0.2-$ $500 \mu \mathrm{g} / \mathrm{ml}$ at $\mathrm{R}^{2}>0.9999$; the intraday and interday precision was determined as relative standard deviation of the peak area and retention time that was found to be $(0.6 \pm 0.4) \%$ and $(0.6 \pm 0.3) \%$, respectively; the percentage recovery for madecassoside, asiaticoside, madecassic acid and asiatic acid were in the range $(95.66 \pm 3.0) \%-(96.04 \pm 4.3) \%,(91.77 \pm 4.7) \%-(100.85 \pm 5.6) \%$, $(98.09 \pm 5.3) \%-(106.38 \pm 2.9) \%$ and $(93.83 \pm 4.9) \%-(95.97 \pm 4.0) \%$; the limits of detection were $0.28-0.29 \mu \mathrm{g} / \mathrm{ml}, 0.85-0.88 \mu \mathrm{g} / \mathrm{ml}$, $0.09-0.10 \mu \mathrm{g} / \mathrm{ml}$ and $0.27-0.29 \mu \mathrm{g} / \mathrm{ml}$, and the limits of quantification were $0.14-0.15 \mu \mathrm{g} / \mathrm{ml}, 0.41-0.44 \mu \mathrm{g} / \mathrm{ml}, 0.19-0.22$ $\mu \mathrm{g} / \mathrm{ml}$ and $0.58-0.65 \mu \mathrm{g} / \mathrm{ml}$, respectively. The reported method was successfully applied for determination of the 4 marker compounds in 15 extracts of $C$. asiatica, and it could be considered as an important analytical tool for quality control, stability studies, pharmacokinetics, and standardization purposes. Preliminary study showed the correlation between principal component analysis (PCA) on the FTIR spectra and the chemical constituents. The extracts that have similar functional group can be clustered together in the same position.

Keywords: RP-HPLC, asiaticoside, asiatic acid, madecassoside and madecassic acid, Centella asiatica. 